\title{
Dividing Connected Chores Fairly ${ }^{\text {th }}$
}

\author{
Sandy Heydrich ${ }^{\mathrm{a}, \mathrm{b}}$, Rob van Stee $\mathrm{c}^{\mathrm{c} 1}$ \\ ${ }^{a}$ Max Planck Institute for Informatics, Saarbrücken, Germany \\ ${ }^{b}$ Saarbrücken Graduate School of Computer Science, Germany \\ ${ }^{c}$ University of Leicester, Leicester, UK
}

\begin{abstract}
In this paper we consider the fair division of chores (tasks that need to be performed by agents, with negative utility for them), and study the loss in social welfare due to fairness. Previous work has been done on this so-called price of fairness, concerning fair division of cakes and chores with non-connected pieces and of cakes with connected pieces. In this paper, we consider situations where each player has to receive one connected piece of the chores. We provide tight or nearly tight bounds on the price of fairness with respect to the three main fairness criteria proportionality, envy-freeness and equitability and for utilitarian and egalitarian welfare. We also give the first proof of the existence of equitable divisions for chores with connected pieces.
\end{abstract}

Keywords: division of chores, price of fairness, cake cutting

\section{Introduction}

Fair division is a topic that has been worked on for millennia. It is known that already the ancient Greeks knew the "cut and choose" method for achieving a fair division of some good between two people (see e.g. [11]). Motivated by the fact that social interaction often requires dividing goods, researchers in economics, law and computer science dealt with this subject since the 1940's. In fair division, one tries to divide some good between a number of people that all have individual preferences and dislikes, while satisfying some fairness condition. We will only focus on the case where the goods are divisible, i.e. can be cut in arbitrary pieces; dividing indivisible goods fairly is a much harder problem. For the setting where each of the players wants to get as much of the goods as possible, one often uses the analogy of cake cutting [16], meaning that we want to divide a cake that has various sections with different toppings. While in cake cutting we want to maximize the happiness of the players with the fraction of the cake they receive, in the chore division [10] or dirty work problem [15, p. 73] one tries to minimize the discontent of the players when dividing work or other disliked goods. Many algorithms found for cake cutting also apply to the division of chores, but interestingly, as we will see in this work their theoretical properties differ in several cases.

Of course one has to decide how to define fairness, and the three criteria proportionality, envy-freeness and equitability considered in many earlier papers (e.g. $[15,1,6])$ will also be considered here. Informal definitions for these are given in the next paragraph. Apart from achieving a division which is fair in some sense, another goal is optimizing the welfare of the division, i.e. to maximize the utility or minimize the disutility of the players. As those two quality criteria for divisions are somehow orthogonal, the natural question arises what the trade-off between those two goals is. Caragiannis et al. [6] and Aumann and Dombb [1] examined this trade-off for the division of cakes and chores; Caragiannis et al. found upper and lower bounds for this trade-off, called the price of fairness, for both cakes and chores, but without any restriction on the number of pieces each player receives. This may lead to the undesirable situation that players receive a huge number of small pieces, e.g. a bunch of crumbs in the cake analogy. Therefore, Aumann and Dombb [1] examined the price of fairness for connected pieces, requiring that every player receive exactly one connected part of the cake; however, they did not consider division of chores. To close the gap, in this paper

\footnotetext{
${ }^{2}$ A preliminary version of this paper appeared in Proc. 6th Intl. Symp. on Algorithmic Game Theory (SAGT 2013), p. 171-182.

Email addresses: heydrich@mpi-inf.mpg.de (Sandy Heydrich), rvs $4 @$ le.ac.uk (Rob van Stee)

${ }^{1}$ Work performed while this author was at Max Planck Institute for Informatics, Saarbrücken, Germany
} 
we give bounds on the price of fairness with connected pieces in division of chores. An analogy for this could be that a group of gardeners needs to maintain a garden and each of them wants to be responsible for one connected area.

\subsection{Model}

In our model, the chores are represented by the real interval $[0,1]$ and we consider $n$ players. Each player has a disutility function over this interval that gives his discontent for a particular piece. These functions are required to be non-atomic measures, i.e. they are non-negative and additive and if an interval is valued strictly positive, it must have a subinterval that has a strictly less but still strictly positive value; furthermore the functions are normalized, so the disutility for the whole chores is 1 . The disutility of a player in a division is then the disutility of this player for the piece he receives. The utilitarian welfare for a division is defined as the sum over the disutilities of all players, while egalitarian welfare is the greatest disutility among all players (i.e. the disutility of the worst-off player). A division is called optimal if it minimizes the welfare. We call a division proportional if every player's disutility is at most $\frac{1}{n}$, we call it envy-free if no player thinks that another player receives less chores than him, and we call it equitable if the disutilities of all players in the division are equal.

To quantify the loss in welfare due to fairness we use the notion of the price of fairness. We define the price of proportionality (respectively envy-freeness, equitability) as the ratio between the welfare of the best proportional (respectively envy-free, equitable) and the welfare of the optimal division.

\subsection{Related Work}

Modern mathematicians started working on the topic in the 1940's with Banach, Steinhaus and Knaster giving the "Last Diminisher" mechanism for proportional divisions with $n$ players [16]. In the following years, research mainly focused on finding algorithms for achieving fair divisions ([4, 5, 9, 17]), also trying to bound the number of cuts required. Furthermore, Dubins and Spanier as well as Stromquist gave existence theorems for certain fair divisions $[9,17]$.

On the problem of fair division of chores however, much less work has been done. The problem was first mentioned by Gardner [10], Oskui [15, p. 73] gave the first three person envy-free chore division algorithm. Peterson and Su gave a four-person envy-free protocol [13] and an $n$ person protocol [14]. The result of Steinhaus [16] can be applied to chores too, so proportional divisions always exist. By Su [18], it is proven that envy-free divisions of chores with connected pieces also always exist. For the existence of equitable divisions with connected pieces, as far as we know no constructive proof was given so far.

The problem of the efficiency of fair divisions was first addressed by Caragiannis et al. [6]. Their work considered the price of fairness for utilitarian welfare and the three fairness notions proportionality, envy-freeness and equitability, and examined bounds for these for divisible and indivisible cakes as well as chores. Aumann and Dombb [1] gave bounds for the price of fairness for utilitarian and egalitarian welfare, restricted to the case that only connected pieces are allowed to be given to the players so they do not end up with "a countable union of crumbs" ([17]), but they only considered cake cutting. In this work we try to find bounds for the remaining case of chore division with connected pieces.

Following the work of Caragiannis et al. and Aumann and Dombb, Cohler et al. [8] provided a polynomial time approximation scheme for computing envy-free cake divisions that are optimal w.r.t. utilitarian welfare. Based on this work, Bei et al. [2] give a polynomial time approximation scheme for computing optimal proportional cake divisions with connected pieces.

Brams et al. [3] connected the topic of efficient fair divisions with the sphere of Pareto-optimal divisions, i.e. divisions in which it is not possible to give one player a strictly higher utility while giving no player a lower utility. They examined whether we can always find fair divisions maximizing the (utilitarian) social welfare that are also Paretooptimal and showed that for a special class of evaluation functions, the optimal (w.r.t utilitarian welfare) equitable division has never a higher social welfare than the optimal envy-free division. However, they did not take connected pieces into account.

\subsection{Overview of Results}

We examine the price of fairness for utilitarian and egalitarian welfare and the three fairness notions proportionality, envy-freeness and equitability as a function of the number of players $n$. We give tight bounds for all cases except 
Table 1: Results of this work, compared to [6] and [1]. Some results only hold for $n \geq 3$. See the text for the case $n=2$.

\begin{tabular}{|c|c|c|c|c|c|}
\hline \multirow[t]{3}{*}{ Chores: } & \multicolumn{3}{|c|}{ connected (this work) } & \multirow{2}{*}{\multicolumn{2}{|c|}{$\frac{\text { non-connected }([6])}{\text { utilitarian }}$}} \\
\hline & \multicolumn{2}{|c|}{ utilitarian } & \multirow[t]{2}{*}{ egalitarian } & & \\
\hline & lower & upper & & lower & upper \\
\hline Proportionality & $n / 2$ & $n$ & 1 & $\frac{(n+1)^{2}}{4 n}$ & $n$ \\
\hline Envy-Freeness & \multicolumn{3}{|c|}{$\infty$} & $\frac{(n+1)^{2}}{4 n}$ & $\infty$ \\
\hline Equitability & & $n$ & 1 & & \\
\hline \multirow[t]{3}{*}{ Cakes: } & \multicolumn{3}{|c|}{ connected ([1]) } & \multirow{2}{*}{\multicolumn{2}{|c|}{$\frac{\text { non-connected }([6])}{\text { utilitarian }}$}} \\
\hline & \multicolumn{2}{|c|}{ utilitarian } & egalitarian & & \\
\hline & lower & upper & & lower & upper \\
\hline Proportionality & $\frac{\sqrt{n}}{2}$ & $\frac{\sqrt{n}}{2}+1-o(1)$ & 1 & $\Omega(\sqrt{n})$ & $O(\sqrt{n})$ \\
\hline Envy-Freeness & $\frac{\sqrt{n}}{2}$ & $\frac{\sqrt{n}}{2}+1-o(1)$ & $n / 2$ & $\Omega(\sqrt{n})$ & $n-1 / 2$ \\
\hline Equitability & $n-1+1 / n$ & $n$ & 1 & $\frac{(n+1)^{2}}{4 n}$ & $n$ \\
\hline
\end{tabular}

for the utilitarian price of proportionality, where there is still a small gap between the lower and the upper bound. All results are summarized and compared to the results by Caragiannis et al. [6] and Aumann and Dombb [1] in Table 1. Essentially the same results, but with a tight bound of $n$ for the utilitarian price of proportionality, were achieved independently by Hoffmann et al. [12].

\subsubsection{The Price of Proportionality}

For utilitarian welfare, we show that the price of proportionality is linear in the number of players. To be precise, we give a lower bound of $n / 2$ and an upper bound of $n$ for $n>2$. The proof for the upper bound is identical to the one given by Caragiannis et al. [6]. For $n=2$ we show a tight bound of 2 . The bounds that were given by Aumann and Dombb [1] for cake cutting with connected pieces are in $\Theta(\sqrt{n})$, Caragiannis et al. [6] found bounds in $\Theta(n)$ for chore division with non-connected pieces.

For egalitarian welfare we have a price of proportionality of 1 for all $n$, meaning that there is no trade-off between proportionality and egalitarian welfare. This is the same result as shown by Aumann and Dombb [1].

\subsubsection{The Price of Envy-Freeness}

While for cake cutting with connected pieces Aumann and Dombb [1] found almost tight bounds in $\Theta(\sqrt{n})$ for utilitarian welfare and the tight bound $n / 2$ for egalitarian welfare, we can show that for the division of chores with more than 2 players and both welfare functions, we can construct instances that have an arbitrarily high price of fairness, i.e. the price of envy-freeness is unbounded. So we see that for this fairness notion there is an inherent difference between cakes and chores and between the connected and the non-connected case. Only for $n=2$ we can derive bounds of 2 (utilitarian welfare) and 1 (egalitarian welfare).

\subsubsection{The Price of Equitability}

As far as we know, our proof is the first constructive proof for the existence of equitable egalitarian-optimal divisions of chores with connected pieces. I.e. we prove that for all $n$ there always exists an equitable division that is as good as the optimal division in terms of egalitarian welfare, in other words the egalitarian price of equitability is 1. We show this by constructing an equitable division starting with an egalitarian-optimal division without increasing any player's disutility above the egalitarian welfare. We also give a tight bound of $n$ for the utilitarian price of equitability for all $n$, by giving an instance of the chore division problem where at least one player has to get a disutility of $\frac{1}{(n-1)^{2}+1}-2 \epsilon$ in every division. For this price of fairness, Aumann and Dombb [1] gave an upper bound of $n$ and a lower bound of $n-1+\frac{1}{n}$, and for non-connected chores, Caragiannis et al. [6] give also a tight bound of $n$. 


\section{Definitions}

In this section we formally define the chores division problem itself, the notions of fairness and social welfare used in this work and finally the price of fairness, the measure for the trade-off between fairness and social welfare.

The real interval $[0,1]$ represents the chores we want to divide. Our players are simply denoted by the numbers $1, \ldots, n$, we write $[n]=\{1, \ldots, n\}$. Each player $i$ has a certain valuation function $v_{i}(\cdot)$, that maps any possible subset of the chores to a real valuation between 0 and 1 . This valuation function needs to be a non-atomic measure with $v_{i}(0,1)=1$.

Definition 1. A division $x$ of the chores is a vector $x=\left(x_{1}, \ldots, x_{n-1}, \pi\right) \in[0,1]^{n-1} \times S_{n}$. The point $x_{i}$ denotes the position of the $i$-th cut, we define $x_{0}:=0, x_{n}:=1$, and the cuts are sorted: $x_{0} \leq x_{1} \leq \ldots \leq x_{n-1} \leq x_{n}$. $\pi$ is a permutation that denotes the assignment of the pieces to the players: Player $i$ receives the interval $\left(x_{\pi(i)-1}, x_{\pi(i)}\right)$. By $X$ we denote the set of all possible divisions.

The unhappiness of the players with a certain division is given by the notion of disutility.

Definition 2. The disutility of a division $x$ for a player $i$ is $d_{i}(x)=v_{i}\left(x_{\pi(i)-1}, x_{\pi(i)}\right)$.

In this work, three different notions of fairness are considered, given in the following definitions.

Definition 3. A division $x$ is proportional if $d_{i}(x) \leq \frac{1}{n}$ for every player $i$.

Intuitively, a division is proportional if all players get a portion they consider their fair share of the chores (or less).

Definition 4. A division $x$ is envy-free if $v_{i}\left(x_{\pi(i)-1}, x_{\pi(i)}\right) \leq v_{i}\left(x_{\pi(j)-1}, x_{\pi(j)}\right)$ for every pair of players $i, j$.

Intuitively, a division is envy-free if no player envies any other player, in the sense that he values the other player's piece less than his own piece. Note that every envy-free division is also proportional.

Definition 5. A division $x$ is equitable if $d_{i}(x)=d_{j}(x)$ for every pair of players $i, j$.

So a division is equitable if the disutilities of all players are equal (by their own valuations).

The social welfare of a division can be defined in two ways:

Definition 6. A division $x$ has utilitarian social welfare $u(x)=\sum_{i \in[n]} d_{i}(x)$.

Definition 7. A division $x$ has egalitarian social welfare eg $(x)=\max _{i \in[n]} d_{i}(x)$.

So in utilitarian welfare, the total disutility of all players is considered, whereas egalitarian welfare refers to the disutility of the worst-off player.

To quantify the amount of social welfare one has to sacrifice to achieve fairness, we define the price of fairness:

Definition 8. The price of fairness (price of proportionality, respectively envy-freeness, equitability) is the minimal welfare achievable in fair (proportional, respectively envy-free, equitable) divisions divided by the minimal welfare achievable in arbitrary divisions.

For example the price of envy-freeness with egalitarian welfare is $\frac{\min _{x \in X_{E F}} e g(x)}{\min _{x \in X} e g(x)}$, where $X_{E F}$ denotes the set of all connected envy-free divisions.

\section{The Price of Proportionality}

We start with bounds for the price of proportionality. For utilitarian welfare, the results do not differ much from the results for non-connected chores by Caragiannis et al. [6], although the lower bound is slightly better. Concerning egalitarian welfare, we can use a proof analogous to the proof by Aumann and Dombb for the price of proportionality with connected cakes [1].

The idea of the lower bound proof for utilitarian welfare is to construct an instance where one player, who is indifferent and dislikes the chores uniformly, receives a piece slightly greater than $\frac{1}{n}$ in the optimal division, and where it is very costly to give some part of this piece to any other player. Intuitively one could say that this is a scenario where one player "sacrifices" himself to do more work than his fair share (in terms of proportionality) would be, as he himself does not dislike this work as much as the other players. 


\subsection{Utilitarian Welfare}

Theorem 1. The utilitarian price of proportionality for the division of chores with connected pieces is lower-bounded by $\frac{n}{2}$ for $n>2$.

Proof. Consider the following instance of the chores division problem: Let $0<\epsilon<\frac{1}{4}-\frac{1}{2 n}$ be arbitrarily small. Players $1, \ldots, n-1$ value the piece $\left(\frac{n-1}{n}-\epsilon, \frac{n-1}{n}\right)$ as $\frac{1}{2}-\epsilon$ and the piece $(1-\epsilon, 1)$ as $\frac{1}{2}+\epsilon$. Player $n$ values the entire chores uniformly. This instance together with its optimal division is shown in figure 1 .

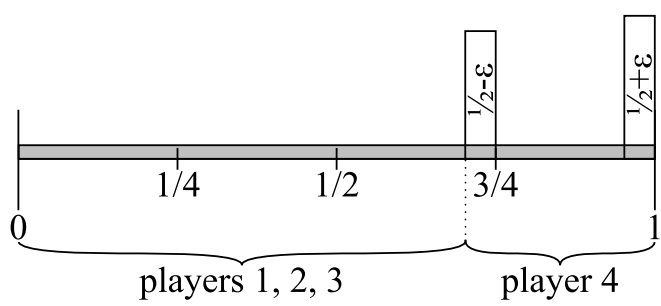

Figure 1: Example construction for $n=4$ players. The two bars at $(3 / 4-\epsilon, 3 / 4)$ and $(1-\epsilon, 1)$ denote the valuations of players $1, \ldots, n-1$. The last player dislikes the whole chores uniformly, marked by a grey bar. Below the interval the optimal division is shown.

In an optimal division, the piece $\left(0, \frac{n-1}{n}-\epsilon\right)$ is assigned to the players $1, \ldots, n-1$ and the rest of the chores to player $n$. This gives 0 disutility for the first $n-1$ players and a disutility of $\frac{1}{n}+\epsilon$ for player $n$, so the utilitarian welfare in such an optimal division is $\frac{1}{n}+\epsilon$. Assigning one of the intervals $\left(\frac{n-1}{n}-\epsilon, \frac{n-1}{n}\right)$ and $(1-\epsilon, 1)$ to one of the other players would cause a greater disutility, for $\epsilon<\frac{1}{4}-\frac{1}{2 n}$ and $n>2$ implies $\frac{1}{n}+\epsilon<\frac{1}{2}-\epsilon$.

In a proportional division however, $n$ cannot be assigned more than $\frac{1}{n}$ of the chores and therefore the intervals at $\left(\frac{n-1}{n}-\epsilon, \frac{n-1}{n}\right)$ and $(1-\epsilon, 1)$ cannot completely be given to player $n$. To be more precise, the other players have to receive fractions of the two intervals $\left(\frac{n-1}{n}-\epsilon, \frac{n-1}{n}\right)$ and $(1-\epsilon, 1)$ of total length $\epsilon$. To achieve an optimal proportional division we assign player $n$ the interval $(1-\epsilon, 1)$ (that is, we give this player the most costly part of the chores w.r.t the other players' disutilities), which gives him a disutility of $\epsilon$ and the rest of the chores is divided between the other players such that the division is proportional (which is possible as $(n-1) \cdot \frac{1}{n} \geq \frac{1}{2}-\epsilon$ for $n>2$ ). Their total disutility is therefore $\frac{1}{2}-\epsilon$. Hence, the utilitarian welfare in this division is $\epsilon+\frac{1}{2}-\epsilon=\frac{1}{2}$.

Finally, the utilitarian price of proportionality is therefore lower-bounded by $\frac{\frac{1}{2}}{\frac{1}{n}+\epsilon}$, which is arbitrarily close to $\frac{n}{2}$ for sufficiently small $\epsilon$.

Note that for this problem instance, it is essential that players have to receive connected pieces of the chores; otherwise we could give only the two strongly disliked pieces $\left(\frac{n-1}{n}-\epsilon, \frac{n-1}{n}\right)$ and $(1-\epsilon, 1)$ to player $n$.

For two players, we consider a problem instance that we will also use in some later proofs: one player is indifferent on the chores, the other dislikes only a small part in the middle. Dividing this instance fairly (for all three fairness measures considered here) always results in giving the indifferent player slightly more than one half of the chores, so that the other player does not receive any part of the chores he dislikes. This again means that we sacrifice proportionality in order to give the indifferent player more work while pleasing the other player.

Theorem 2. The utilitarian price of proportionality for $n=2$ players is lower-bounded by 2 .

Proof. Consider the following instance: Player 1 values the piece $\left(\frac{1}{2}-\epsilon, \frac{1}{2}+\epsilon\right)$ as 1 and the rest as 0 , while player 2 values the entire interval uniformly. In an optimal division, we give player 1 the piece $\left(0, \frac{1}{2}-\epsilon\right)$ and player 2 the piece $\left(\frac{1}{2}-\epsilon, 1\right)$. The utilitarian welfare of such a division is $\frac{1}{2}+\epsilon$, the physical size of the piece of player 2 . To achieve a utilitarian-optimal proportional division, we give each player exactly one half of the chores (note that this division is also envy-free and equitable), which gives both a disutility of $\frac{1}{2}$ and a utilitarian welfare of 1 . Hence the price of fairness is arbitrarily close to $\frac{1}{1 / 2}=2$ for sufficiently small $\epsilon$.

For the upper bound on the utilitarian price of proportionality, we can cite the proof by Caragiannis et al. [6], as it also applies to connected chores. 
Theorem 3. The utilitarian price of proportionality for the division of chores with connected pieces is upper-bounded by $n$.

Proof. Let an arbitrary instance of the chores division problem be given, let $x$ be an optimal and $y$ a proportional optimal allocation for this instance. Then either $x$ is already proportional - in this case the price of proportionality is 1 - or at least one player has a disutility $>\frac{1}{n}$. In this case, also the total (utilitarian) disutility of $x$ is $>\frac{1}{n}$. In $y$ however, any player's disutility is at most $\frac{1}{n}$ by definition of proportionality. Thus the utilitarian welfare of $y$ is at most 1 , and the price of proportionality is upper-bounded by $\frac{1}{1 / n}=n$.

\subsection{Egalitarian Welfare}

Completely analogous to Aumann and Dombb [1], we prove that every egalitarian-optimal division is also proportional, hence we do not have to give up proportionality to achieve egalitarian-optimal divisions in any instance.

Theorem 4. Every egalitarian-optimal division of chores with connected pieces is proportional, and therefore the egalitarian price of proportionality in this case is 1 .

Proof. Let again an arbitrary instance of the chores division problem be given with an optimal division $x$ and an arbitrary proportional division $y$. By definition of proportionality we know that $d_{i}(y) \leq \frac{1}{n}$ for all players $i$, hence also $e g(y)=\max _{i \in[n]} d_{i}(y) \leq \frac{1}{n}$. By definition of egalitarian welfare, for all players $i, d_{i}(x) \leq e g(x)$ and as $x$ is egalitarian-optimal, we know $d_{i}(x) \leq e g(x) \leq e g(y) \leq \frac{1}{n}$, i.e. $x$ is proportional.

\section{The Price of Equitability}

The price of equitability is a more interesting case than proportionality, as so far no constructive proof was given for the existence of equitable divisions of chores with connected pieces. The existence proof of this kind of division for cakes was given by Aumann and Dombb [1]. With some minor changes, their proof might actually be carried over to our setting:

- We start with an egalitarian-optimal division with egalitarian welfare $O P T$. First, we need to move cuts between the first $n-1$ pieces to the right until they all have the value $O P T$. However, in constrast to the cake cutting scenario, it might be not possible to move all these cuts to the right as far as necessary. If some cut reaches the boundary, we stop this process and all following pieces are empty.

- In the second step, we need to move the last cut to the left until the last piece has value $O P T-\epsilon$. Aumann and Dombb only need to move this one cut to the right until the last piece has value $O P T+\epsilon$, however, in our setting it might be necessary to also move other cuts if the rightmost cut touches them, and therefore pieces of other players might get empty. But the valuations for all players except the last one are adjusted in a recursive step, and additionally we know by optimality of the original division that at least one piece will stay at value $O P T$. Therefore, Aumann and Dombb's further argumentation carries over.

This approach by Aumann and Dombb, however, is strictly speaking not constructive. They show how to construct an egalitarian-optimal division in which no player's utility deviates from the optimum by more than $\epsilon$ for any $\epsilon>0$ (you could say "nearly-equitable divisions"). The existence of an egalitarian-optimal equitable division then follows from the compactness of the set of all egalitarian-optimal divisions. During the work on this paper, another existence proof for such cake divisions was given by Cechlárová, Dobos and Pillárová [7] (they even prove a stronger claim), but their proof is non-constructive as well. We complete the picture by giving a constructive existence proof for equitable chore divisions with connected pieces that are egalitarian-optimal.

In Theorem 5, we show how to transform any egalitarian-optimal division into an equitable one with the same welfare. The construction relies on the fact that optimality with respect to egalitarian welfare and the non-atomicity of the evaluation measures imply that we can make pieces that are adjacent to a piece with maximal value (among all pieces) also maximal.

Afterwards we give proofs for a tight bound of $n$ for utilitarian welfare. This generalizes the bound for nonconnected chores given by Caragiannis et al. [6]. 


\subsection{Egalitarian welfare}

Theorem 5. For every instance of the chores division problem, there exists an equitable division with connected pieces. Furthermore, the egalitarian price of equitability for the division of chores with connected pieces is 1.

Proof. We need some more terminology for this proof:

- The value of a piece is the value that is assigned to this piece by the player who receives it.

- Let $m=\min _{x \in X} e g(x)$ be the optimal egalitarian welfare. Pieces that have a value of $m$ are called maximal pieces.

- We call a sequence of one or more adjacent maximal pieces $p_{1}, \ldots, p_{k}$ a block of maximal pieces, if

- the left neighbour of $p_{1}$ is non-maximal or $p_{1}$ is the left-most piece of the division, and

- the right neighbour of $p_{k}$ is non-maximal or $p_{k}$ is the right-most piece of the division

We call the left neighbour of $p_{1}$ and the right neighbour of $p_{k}$ the neighbours of this block (if they exist).

Consider an egalitarian-optimal division $x$ that has the minimal number of maximal pieces among all egalitarianoptimal divisions. We want to make all pieces in $x$ maximal by moving cuts, and for this we first show a lemma:

Lemma 1. Consider a division $x$ with a block $p_{1}, \ldots, p_{k}$ of $k$ maximal pieces that has a right neighbor $p^{\prime}$ (i.e. the rightmost piece of the block is not the last piece). Let l be the left border of the block, $r$ the right border and $r^{\prime}$ the right border of $p^{\prime}$. Then there exists a division $x^{\prime}$ which is identical to $x$ on $[0, l]$ and $\left[r^{\prime}, 1\right]$, and which has one of the following two properties.

(a) The pieces $p_{1}, \ldots, p_{k}$ and $p^{\prime}$ are all maximal.

(b) None of the pieces $p_{1}, \ldots, p_{k}$ and $p^{\prime}$ are maximal.

Proof. We do an induction on the number of pieces in the block $k$ :

Induction base: $k=1$. We move the cut between our only maximal piece $p$ and its right neighbour $p^{\prime}$ to the left, i.e. we make $p$ physically smaller and $p^{\prime}$ physically larger. Either at some point the value of $p$ falls below $m$, then our whole block is non-maximal, or eventually $p^{\prime}$ becomes maximal.

Induction step: $k-1 \rightarrow k$. Move $r$ to the left until $p^{\prime}$ is maximal (stop at the first point where $p^{\prime}$ is maximal). If this is not possible, we can make the whole block non-maximal (we move $r$ to the left until it reaches $l$; then all pieces of the block are empty). So assume, $p^{\prime}$ becomes maximal when $r$ reaches some point $r^{*}>l$. If $p_{k}$ is still maximal, we are done. Otherwise, the pieces to the left of $p_{k}$ are a block of size at most $k-1$, hence we can apply the induction hypothesis. Then either we can make $p_{k}$ (and all pieces to the left of $p_{k}$ that became non-maximal) maximal again by only moving cuts inside the block; in particular, we do not change $r$ and therefore $p^{\prime}$ is still maximal. Or it is now possible to make all $k-1$ pieces non-maximal. Then the block including $p_{k}$ is non-maximal, and $p^{\prime}$ is maximal. Say $p_{k}$ 's value is $m-\epsilon$ for some $\epsilon>0$. As the valuation functions are non-atomic measures, there must exist a $\delta>0$, such that moving $r$ back to the right by $\delta$ increases $p_{k}$ by less than $\epsilon$. On the other hand, we moved $r$ only as far to the left as needed so that $p^{\prime}$ became maximal, hence moving $r$ to the right by $\delta$ decreases $p^{\prime}$ 's value below $m$. Therefore, all pieces of the original block and $p^{\prime}$ are non-maximal.

The same lemma can also be shown for the left neighbour completely symmetrically.

Now consider our optimal division $x$ and look at its left-most block of maximal pieces. By our lemma we can make its right neighbour maximal, as otherwise the block would become non-maximal and this would contradict the assumption that $x$ is the division with minimal number of maximal pieces. But by applying the lemma again and again, we can make all pieces to the right of this (steadily growing) left-most block maximal. Note that every time, if we find that we can make the entire block non-maximal, we find a division with less maximal pieces than $x$, which is a contradiction. If we reach a piece that is already maximal during this process, we just add it to the block without moving cuts of course. We can then apply the lemma symmetrically for the pieces to the left of this block (which is now the only block of maximal pieces in $x$ ) and make them all maximal too. Finally we have a division where all pieces are maximal. 


\subsection{Utilitarian welfare}

While achieving equitability does not influence the egalitarian optimality, it has a huge impact on the utilitarian welfare, as shown in the next three theorems. The idea of the lower bound proof is to make sure that one indifferent player has to receive at least a piece of a certain value in both fair and unfair divisions, which leads to a price of fairness of $n$, as in equitable divisions all player have to receive this certain disutility, while the indifferent player is the only one to receive any disliked piece in the utilitarian-optimal division.

Theorem 6. The utilitarian price of equitability is lower-bounded by $n$ for $n>2$ players.

Proof. We construct an instance of the chores division problem that has a utilitarian price of equitability of at least $n$ as follows:

Let $\epsilon>0$ be arbitrarily small. We create $(n-1)^{2}$ so-called "disliked pieces" $p_{1}, \ldots, p_{(n-1)^{2}}$, where $p_{i}$ is located at $\left(\frac{i}{(n-1)^{2}+1}-\epsilon, \frac{i}{(n-1)^{2}+1}+\epsilon\right)$.

We divide those pieces into $(n-1)$ blocks of $n-1$ pieces each, and each block contains one piece for every player $\{1, \ldots, n-1\}$. The first piece of the first block is associated with player 1 , the second with player 2 and so on, until the last piece of the first block is associated with player $n-1$. The pieces of the second block are then associated with players $2,3, \ldots, n-1,1$ (in this order), the pieces of the third block with players $3,4, \ldots, n-1,1,2$ and so on. Generally, the pieces of the $i$-th block are associated with players $i, i+1, \ldots, n-1,1, \ldots, i-1$. Each player values each piece associated with him as $\frac{1}{n-1}$ and the rest of the chores as 0 , which sums up to a total valuation of 1 for the entire chores for each of the first $n-1$ players. Finally player $n$ values the entire chores uniformly. This construction is shown in figure 2 .

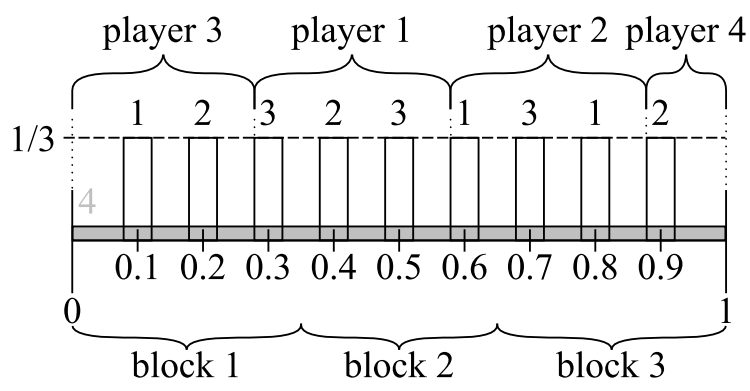

Figure 2: Example construction for $n=4$ players. The numbers above the columns denote the player this piece is associated with. Above the interval the optimal division is shown.

First, we take a look at the optimal division for this instance, that is obtained as follows: we give the piece $\left(0, \frac{n-1}{(n-1)^{2}+1}-\epsilon\right)$ to player $n-1$, the piece $\left(\frac{i \cdot(n-1)}{(n-1)^{2}+1}-\epsilon, \frac{(i+1) \cdot(n-1)}{(n-1)^{2}+1}-\epsilon\right)$ to player $i$ for $i=1, \ldots, n-2$ and finally the piece $\left(\frac{(n-1)^{2}}{(n-1)^{2}+1}-\epsilon, 1\right)$ to player $n$.

We observe the following:

- For $i=1, \ldots, n-2$ the $i$-th disliked piece of player $i$ is at $\left.\frac{(i-1) \cdot(n-1)+1}{(n-1)^{2}+1}-\epsilon, \frac{(i-1) \cdot(n-1)+1}{(n-1)^{2}+1}+\epsilon\right)$. This follows by construction, as this piece is the first piece of the $i$-th block.

- For $i=1, \ldots, n-2$ the $i+1$-st disliked piece of player $i$ is at $\left(\frac{(i+1) \cdot(n-1)}{(n-1)^{2}+1}-\epsilon \frac{(i+1) \cdot(n-1)}{(n-1)^{2}+1}+\epsilon\right)$. This also follows by construction, as this piece is the last piece of the $i+1$-st block.

We conclude that the piece that is assigned to player $i=1, \ldots, n-2$ as above is between the $i$-th and $i+1$-st piece of this player. The piece player $n-1$ receives is before his first disliked piece. Hence, the disutility of the players $1, \ldots, n-1$ are all 0 , as they all do not receive any of their disliked pieces. Player $n$ 's disutility is exactly the physical size of the piece he receives, so the utilitarian welfare in this division is $u(x)=d_{n}(x)=1-\left(\frac{(n-1)^{2}}{(n-1)^{2}+1}-\epsilon\right)=$ $\frac{1}{(n-1)^{2}+1}+\epsilon$. The optimal division for the example with $n=4$ players can be seen in figure 2 . 
Now, we claim that the disutility of player $n$ in any equitable division of the chores is at least $\frac{1}{(n-1)^{2}+1}-2 \epsilon$. From this it follows that the utilitarian welfare in equitable divisions is at least $n \cdot\left(\frac{1}{(n-1)^{2}+1}-2 \epsilon\right)$, as all players must have the same disutility. Hence, the price of equitability is then at least $\frac{\frac{n}{(n-1)^{2}+1}-2 n \epsilon}{\frac{1}{(n-1)^{2}+1}+\epsilon}$. The bound follows as $\epsilon$ approaches 0 .

We first show a lemma:

Lemma 2. There does not exist a division in which player $n$ receives no piece and each other player has disutility 0.

Proof. Assume we could divide the chores between the first $n-1$ players entirely s.t. every player's disutility is 0 . Some player must therefore receive a piece starting at 0 . But this piece cannot cover more than the first block of disliked pieces, as in this block one disliked piece of this player is contained and he cannot receive that. In particular, his piece must end somewhere before his disliked piece and therefore inside the first block. Accordingly, the second piece must end somewhere in the second block and so on. Then, the last piece that is assigned to any of the $n-1$ players must end somewhere in the (last) $n-1$-st block, namely before his last disliked piece. But then the interval from this last disliked piece to the right border of the chores stays unassigned. Contradiction.

So it remains to show that player $n$ 's disutility is always at least $\frac{1}{(n-1)^{2}+1}-2 \epsilon$. First of all, player $n$ cannot have a disutility of 0 , as then he wouldn't receive anything of the chores, leaving the whole chores to players $1, \ldots, n-1$. But as shown in the lemma above, we cannot divide the chores between the first $n-1$ players such that each of them gets 0 disutility, so player $n$ has to receive some piece of the chores.

Assume player $n$ 's disutility is less than $\frac{1}{(n-1)^{2}+1}-2 \epsilon$. That implies that all other players cannot get one of their disliked pieces entirely (as they have a valuation of $\frac{1}{n-1}>\frac{1}{(n-1)^{2}+1}-2 \epsilon$ for each of those pieces), but they have to get fractions of their disliked pieces. There are two cases now:

(a) The piece for player $n$ is the left-most or right-most piece

(b) The piece for player $n$ is somewhere in between the other players' pieces

If player $n$ 's piece is the right-most piece, then accordingly to the argumentation in the proof of Lemma 2 the last piece assigned to any other player must end somewhere in the last block (as otherwise some player would get his entire disliked piece), so player $n$ 's piece must reach from the right border of the interval into the last block, therefore his disutility is greater than $\frac{1}{(n-1)^{2}+1}-\epsilon$ (the size of the slot between the last disliked piece and 1). Note that the argumentation in the proof of Lemma 2 can also be applied "from right to left": Some player receives a piece starting at the right border and then the $i$-th piece from the right must end in block $n-i$. Hence the case when player $n$ receives the left-most piece is symmetric to the case that he receives the right-most piece and we are done for the first case. Consider the other case, when player $n$ 's piece is in between the pieces of the other players. Say there are $i$ players receiving the chores on the left of $n$ 's piece and $n-1-i$ players receiving the chores on the right. Then again the left border of player $n$ 's piece is somewhere in the $i$-th block and the right border somewhere in the $i+1$-st block, hence player $n$ must receive a piece that has a physical size of more than $\frac{1}{(n-1)^{2}+1}-2 \epsilon$, since this is the size of the slot between the last piece of the $i$-th block and the first piece of the $i+1$-st block. 2.

For the special case of two players, the lower bound proof uses the same instance we have seen before in Theorem

Theorem 7. The utilitarian price of equitability for $n=2$ players is lower-bounded by 2.

Proof. Consider the same instance as in the proof of Theorem 2: Player 1 values the piece $\left(\frac{1}{2}-\epsilon, \frac{1}{2}+\epsilon\right)$ as 1 and the rest as 0 , while player 2 values the entire interval uniformly. In an optimal division the utilitarian welfare is $\frac{1}{2}+\epsilon$, and in the optimal equitable division (the same as in Theorem 2), the welfare is 1 . The price of fairness therefore approaches $\frac{1}{1 / 2}=2$ for sufficiently small $\epsilon$.

For proving a matching upper bound, we re-use the constructive proof of Theorem 5 to construct an equitable division which is at most $n$-times as bad as the utilitarian-optimal division.

Theorem 8. The utilitarian price of equitability is upper-bounded by $n$. 
Proof. Consider a chores division instance and let $x$ be a utilitarian-optimal division for it. Of course the egalitarian welfare of $x$ is at most the utilitarian welfare of $x$, i.e. $e g(x) \leq u(x)$. According to Theorem 5 there exists an equitable division $y$ (which we can construct with the method described in the proof of Theorem 5) that achieves the same egalitarian welfare as the egalitarian-optimal division, hence $y$ has at most the same egalitarian welfare as $x$ ( $x$ does not have to be egalitarian-optimal of course). Obviously, $u(y) \leq n \cdot e g(y)$. But then, $u(y) \leq n \cdot e g(y) \leq$ $n \cdot e g(x) \leq n \cdot u(x)$, i.e. the utilitarian price of fairness is at most $n$.

\section{The Price of Envy-Freeness}

Finally, we take a look at the price of envy-freeness. For this fairness notion, we get the most interesting deviation from former results on connected cakes and non-connected chores, as we can prove unboundedness of the price of fairness here (for more than two players). In contrast to the previous theorems, the arbitrary high price of fairness now does not result from giving an indifferent player more than his fair share in the optimal division, which results in high costs when assuring fairness, but from the fact that in the optimal division for the concrete instance given below, it is optimal to give the indifferent player no piece of the chores; however note that this player is now only indifferent on several pieces of the chores, not on the whole chores. But a situation where one player does not receive any piece in the optimal division has a negative effect on the price of envy-freeness, as every other player receiving a disutility $>0$ will envy this player. By choosing the preferences in a certain way, we can make the price of envy-freeness arbitrarily high, as shown below.

Theorem 9. The price of envy-freeness for the division of chores with connected pieces is unbounded for both utilitarian and egalitarian welfare for $n>2$ players.

Proof. We use the same construction we used in the proof of Theorem 6 for the utilitarian price of equitability with other valuation functions, which can have an arbitrarily large price of envy-freeness. Again, let $0<\epsilon<$ $\frac{1}{(n-1)^{2}}$ and consider $(n-1)^{2}$ disliked pieces arranged in $(n-1)$ blocks as before, where piece $p_{i}$ is located at $\left(\frac{i}{(n-1)^{2}+1}-\epsilon, \frac{i}{(n-1)^{2}+1}+\epsilon\right)$ for $i=1, \ldots,(n-1)^{2}$. Call the first piece of each block "type A" piece, the other pieces "type B" pieces.

As before, the pieces of the $i$-th block are associated with players $i, i+1, \ldots, n-1,1, \ldots, i-1$. Each of them values the only type A piece that is associated with him as $1-(n-2) \epsilon$, the $(n-2)$ type B pieces associated with him as $\epsilon$ and the rest of the chores as 0 . Player $n$ assigns a value of $\frac{1}{(n-1)^{2}}$ to every disliked piece of either type and 0 to the rest. An example construction with 4 players can be found in figure 3.
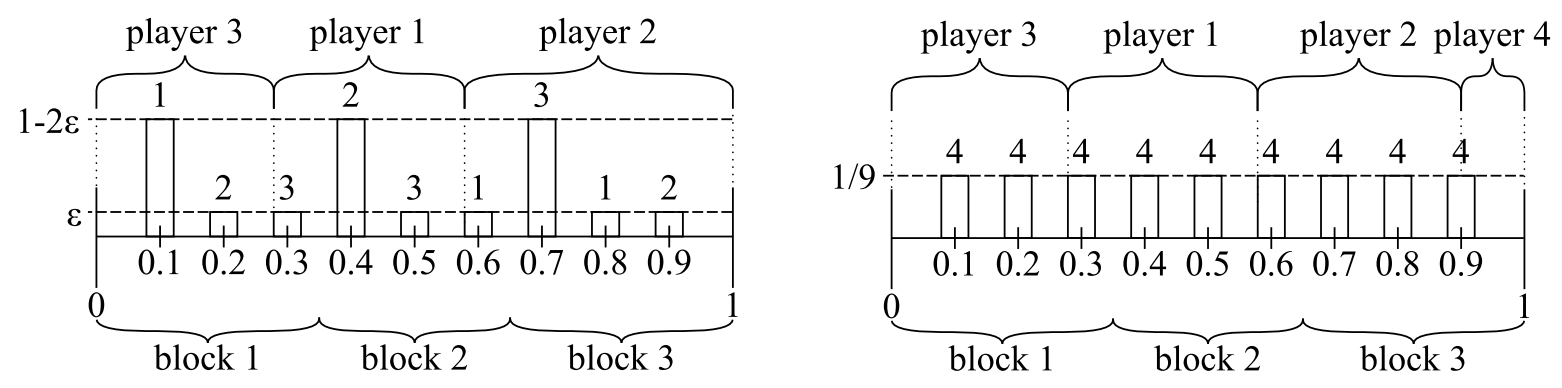

Figure 3: Example construction for $n=4$. For better readability, the left image shows the valuations of players 1 to 3 , the right image shows the valuation of player 4 . The numbers above the columns denote the player this piece is associated with. Above the left interval the optimal division is shown, above the right interval the envy-free division is shown.

An optimal division is constructed as follows: Similarly to the optimal division given in the proof for Theorem 6 , we give the piece $\left(0, \frac{n-1}{(n-1)^{2}+1}-\epsilon\right)$ to player $n-1$, the piece $\left(\frac{i \cdot(n-1)}{(n-1)^{2}+1}-\epsilon \frac{(i+1) \cdot(n-1)}{(n-1)^{2}+1}-\epsilon\right)$ to player $i$ for $i=1, \ldots, n-3$, but we give the whole remaining piece $\left(\frac{(n-2)(n-1)}{(n-1)^{2}+1}-\epsilon, 1\right)$ to player $n-2$. Player $n$ does not receive any piece of the chores. This optimal division is also shown in figure 3.

We observe the same facts as before: 
- For $i=1, \ldots, n-2$ the $i$-th disliked piece of player $i$ is at $\left(\frac{(i-1) \cdot(n-1)+1}{(n-1)^{2}+1}-\epsilon, \frac{(i-1) \cdot(n-1)+1}{(n-1)^{2}+1}+\epsilon\right)$.

- For $i=1, \ldots, n-2$ the $i+1$-st disliked piece of player $i$ is at $\left(\frac{(i+1) \cdot(n-1)}{(n-1)^{2}+1}-\epsilon, \frac{(i+1) \cdot(n-1)}{(n-1)^{2}+1}+\epsilon\right)$.

Therefore, the piece player $i=1, \ldots, n-3$ receives is between the $i$-th and $i+1$-st piece of this player. The piece player $n-1$ receives is before his first disliked piece (which starts at $\frac{n-1}{(n-1)^{2}+1}-\epsilon$ ). Hence, all players except player $n-2$ do not receive any of their disliked pieces and therefore have disutility 0 . Only player $n-2$ receives one type B piece, therefore the maximal disutility, the egalitarian welfare and the utilitarian welfare of the optimal division is $\epsilon$. Note that the optimal division from Theorem 6 yields in this case a higher disutility, namely $\frac{1}{(n-1)^{2}}$ (which is strictly greater than $\epsilon$ according to our assumptions).

This optimal division however is not envy-free, as player $n-2$ envies player $n$, for the empty piece is preferred by every player. Analogous to Lemma 2, we can argue that it is impossible to divide the chores between the first $n-1$ players entirely without giving one player a piece he dislikes. We are always left with at least one disliked piece $p$ that has to be assigned to one player among players $1, \ldots, n-1$ that values it $>0$. Furthermore, this means we also have to give some piece to player $n$, as otherwise all players receiving a disutility $>0$ envy $n$, and assigning $n$ less than one half of $p$ makes the player who receives the rest of $p$ envy $n$. Thus, we can show that we cannot do better than giving $n$ one half of $p$ and another player $i$ who also dislikes $p$ the other half. Therefore, to achieve an optimal envy-free division we give players $1, \ldots, n-3, n-1$ the same pieces as in the optimal division, the piece $\left(\frac{(n-2)(n-1)}{(n-1)^{2}+1}-\epsilon \frac{(n-1)^{2}}{(n-1)^{2}+1}\right)$ to player $n-2$ and the piece $\left(\frac{(n-1)^{2}}{(n-1)^{2}+1}, 1\right)$ to player $n$, i.e. we split the type B piece that player $n-2$ received in the optimal division between players $n-2$ and $n$. In this division $x, d_{n-2}(x)=\frac{\epsilon}{2}$ and $d_{n}(x)=\frac{1}{2(n-1)^{2}}$. As both get the same amount of player $n-2$ 's last disliked piece, $n-2$ does not envy $n$ any more. Furthermore, player $n$ receives half of a disliked piece, whereas every other player receives more than one such piece, hence $n$ does also not envy any other player. Players $1, \ldots, n-3, n-1$ have 0 disutility and therefore also envy no other player. Finally, $d_{n}(x)>d_{n-2}(x)$ for $\epsilon<\frac{1}{(n-1)^{2}}$, hence $e g(x)=d_{n}(x)=\frac{1}{2(n-1)^{2}}$, and $u(x)=d_{n-2}(x)+d_{n}(x)=\frac{\epsilon}{2}+\frac{1}{2(n-1)^{2}}$.

So finally we have a utilitarian price of envy-freeness of $\frac{1}{2}+\frac{1}{2(n-1)^{2} \epsilon}$ and an egalitarian price of envy-freeness of $\frac{1}{2(n-1)^{2} \epsilon}$, which both becomes arbitrarily large when $\epsilon$ approaches 0 .

For the case with two players, we can give tight bounds for both the utilitarian and egalitarian price of envyfreeness, essentially due to the fact that we can switch the two players' pieces for achieving envy-freeness.

Theorem 10. The utilitarian price of envy-freeness for $n=2$ players is 2 .

Proof. First consider the same instance as in the proof of Theorem 2: Player 1 values the piece $\left(\frac{1}{2}-\epsilon, \frac{1}{2}+\epsilon\right)$ as 1 and the rest as 0 , while player 2 values the entire interval uniformly. In an optimal division, the utilitarian welfare is $\frac{1}{2}+\epsilon$, and in the optimal envy-free division (the same as in Theorem 2) the welfare is 1 . Hence the price of fairness is arbitrarily close to 2 for sufficiently small $\epsilon$.

For the upper bound we consider two cases: Either the utilitarian-optimal division has welfare $\leq \frac{1}{2}$, which means that both players must have a disutility of $\leq \frac{1}{2}$. But then in both players' eyes, the piece of the other player has value $1-d_{1}(x) \geq \frac{1}{2}$ respectively $1-d_{2}(x) \geq \frac{1}{2}$, i.e. they both do not envy the other. Therefore, in this case the price of fairness is 1 . So consider the case where the utilitarian-optimal division has welfare $>\frac{1}{2}$. Assume, that the utilitarian welfare in the best envy-free division was $>1$. That would mean that either $d_{1}(x)>\frac{1}{2}$ or $d_{2}(x)>\frac{1}{2}$, but then one of the players would envy the other one and the division would not be envy-free. Therefore, the welfare in utilitarian-optimal envy-free divisions is at most 1 . Thus the price of fairness is at most $\frac{1}{1 / 2}=2$.

Theorem 11. The egalitarian price of envy-freeness for $n=2$ players is 1 .

Proof. If the egalitarian-optimal division has a welfare of $\leq \frac{1}{2}$, we can again argue as in the previous theorem that then this egalitarian-optimal division is also envy-free. Consider therefore optimal divisions with welfare $>\frac{1}{2}$. That means that one of the two players has a disutility of $>\frac{1}{2}$, say it is w.l.o.g. player 1 . But now we can shift our only cut towards the piece of player 1 until his disutility is $\frac{1}{2}$. Now of course the disutility of player 2 could have become (or still be) $>\frac{1}{2}$. But in this case, interchange the pieces the players receive. Player 1 still receives one half of the chores, player 2 receives now less than one half. Therefore, the welfare in this division is $\frac{1}{2}$, a contradiction to the assumption that the best egalitarian welfare in any achievable division is $>\frac{1}{2}$. 


\section{Conclusion}

In this work we examined the decrease of social welfare due to fairness when dividing chores so that every player receives exactly one connected piece of the chores. We considered three important fairness criteria and two different social welfare functions and found tight bounds for almost all cases. For utilitarian welfare and proportionality or equitability the bounds are in $\Theta(n)$, for egalitarian welfare there is no trade-off for these two fairness criteria. For envy-freeness however, no bound exists for both welfare functions except for 2 players.

Upon finding that the price of envy-freeness for the division of chores is the only case that is unbounded, one could ask the question why there is such a fundamental difference between the price of envy-freeness and the price of proportionality or equitability, and why this difference does not appear when considering cakes (Aumann and Dombb [1] gave bounds for this case). To answer the first question, one should note that there is an inherent difference between envy-freeness and the other two fairness notions, namely that the first relies on the valuation of a player for pieces other than his own, while in the latter case we only consider properties of the valuations each player has for his particular piece. The difference between chores and cakes seems to arise from the different nature of the two problems. Infinite envy always results from one player receiving no piece of the cake/chores - then this player is either the one who envies other players (cake division) or the one who is envied by other players (division of chores). Giving one player no chores is - at least in some instances - desirable in chores division, as we have seen in the proof of Theorem 9. When dividing cakes, however, this is neither desirable when considering utilitarian welfare (see Aumann and Dombb [1, Theorem 1]) nor when considering egalitarian welfare, as here the player receiving the least utility determines the amount of welfare. This difference can also be seen in the results for indivisible cakes and chores by Caragiannis et al. [6], where the price of envy-freeness is bounded for cakes and unbounded for chores. It should be noted that a difference between cake and chore division was also shown by Peterson and Su [14]. They translated the $n$-person envy-free cake division protocol of Brams and Taylor [4] to chore division and argued why chore division "is not exactly a dual or straightforward extension of the cake-cutting problem" [14]. Interestingly, their procedure for chore division is more complicated than the cake cutting protocol by Brams and Taylor, but may converge faster and require fewer cuts.

Some questions still remain open. For the setting where non-connected pieces are allowed, Caragiannis et al. [6] only considered utilitarian welfare. It would be interesting to give bounds for the egalitarian welfare function for non-connected divisions of cakes and chores. Furthermore, Caragiannis et al. [6] provided an analysis of the price of fairness for indivisible cakes and chores, but again they only considered utilitarian welfare. Further research could examine the impact of fairness on egalitarian welfare for this setting.

\section{References}

[1] Yonatan Aumann and Yair Dombb. The efficiency of fair division with connected pieces. In Amin Saberi, editor, WINE, volume 6484 of Lecture Notes in Computer Science, pages 26-37. Springer, 2010.

[2] Xiaohui Bei, Ning Chen, Xia Hua, Biaoshuai Tao, and Endong Yang. Optimal proportional cake cutting with connected pieces. In Jörg Hoffmann and Bart Selman, editors, AAAI. AAAI Press, 2012.

[3] Steven Brams, Michal Feldman, John Lai, Jamie Morgenstern, and Ariel Procaccia. On maxsum fair cake divisions. 2012.

[4] Steven J. Brams and Alan D. Taylor. An envy-free cake division protocol. American Mathematical Monthly, 102(1):9-18, January 1995.

[5] Steven J. Brams, Alan D. Taylor, and William Zwicker. A moving-knife solution to the four-person envy-free cake division. In Proceedings of the American Mathematical Society, volume 125, pages 547-554, 1997.

[6] Ioannis Caragiannis, Christos Kaklamanis, Panagiotis Kanellopoulos, and Maria Kyropoulou. The efficiency of fair division. In Stefano Leonardi, editor, WINE, volume 5929 of Lecture Notes in Computer Science, pages 475-482. Springer, 2009. 
[7] Katarína Cechlárová, Jozef Dobos, and Eva Pillárová. On the existence of equitable cake divisions. Inf. Sci., 228:239-245, 2013.

[8] Yuga J. Cohler, John K. Lai, David C. Parkes, and Ariel D. Procaccia. Optimal envy-free cake cutting. In Proceedings of the Twenty-Fifth AAAI Conference on Artificial Intelligence, 2011.

[9] L. E. Dubins and E. H. Spanier. How to cut a cake fairly. American Mathematical Monthly, 68(1):1-17, 1961.

[10] Martin Gardner. Aha! Insight. W. F. Freeman and Co., 1978.

[11] Hesiod. Theogony.

[12] Yosef Hoffman, Yonatan Aumann, and Yair Dombb. Private communication.

[13] Elisha Peterson and Francis E. Su. Four-person envy-free chore division. Mathematics Magazine, 75(2):117-122, 2002.

[14] Elisha Peterson and Francis E. Su. N-person envy-free chore division. arXiv.org:0909.0303, 2009.

[15] Jack M. Robertson and William A. Webb. Cake-cutting algorithms - be fair if you can. A K Peters, 1998.

[16] Hugo Steinhaus. The problem of fair division. Econometrica, 16:101-104, 1948.

[17] Walter Stromquist. How to cut a cake fairly. American Mathematical Monthly, 87(8):640-644, 1980.

[18] Francis E. Su. Rental harmony: Sperner's lemma in fair division. American Mathematical Monthly, 106(10):930$942,1999$. 